\title{
COMPETÊNCIAS E HABILIDADES DA INDÚSTRIA 4.0 NO CONTEXTO DOS CURSOS DE ADMINISTRAÇÃO E COMÉRCIO EXTERIOR
}

\author{
Julio Cesar Zilli \\ Universidade do Extremo Sul Catarinense \\ zilli42@hotmail.com \\ André Luís Silvério Leopoldo \\ Universidade do Extremo Sul Catarinense \\ andreluizh7@gmail.com \\ Patrícia de Sá Freire \\ Universidade Federal de Santa Catarina \\ patriciadesafreire@gmail.com \\ Adriana Carvalho Pinto Vieira \\ Universidade Federal de Mato Grosso \\ dricpvieira@gmail.com \\ Estela Boiani \\ Universidade Federal de Santa Catarina \\ estelaboiani.arq@gmail.com
}

\begin{abstract}
Resumo
Este estudo objetivou identificar a percepção dos acadêmicos dos cursos de Administração e Comércio Exterior da Unesc, diante das suas competências e habilidades frente àquelas requeridas pela Indústria 4.0. Caracterizou-se como uma pesquisa quantitativa, descritiva, de cunho bibliográfico e de campo. A população foi composta por 203 acadêmicos, com a participação efetiva de 80 que responderam um questionário. Optou-se pela frequência simples com vínculo a literatura. Os acadêmicos concordam com o fato de que a quarta revolução criará uma produção mais precisa e personalizável, e também uma nova demanda de profissionais, mas discordam do fato de que os modelos atuais de educação e capacitação precisam de atualização. Os impactos na profissão do Administrador são conhecidos pelos acadêmicos, e os mesmos sabem quais profissões serão as mais afetadas. Por fim, percebese que os acadêmicos possuem em parte nos seus perfis, as competências e habilidades necessárias à Indústria 4.0.
\end{abstract}

Palavras-chave: Competências. Habilidades. Indústria 4.0. Revolução.

\section{COMPETENCIES AND SKILLS OF THE INDUSTRY 4.0 IN THE CONTEXT OF ADMINISTRATION AND FOREIGN TRADE COURSES}

\begin{abstract}
This study aimed to identify the perception of academics in the courses of Administration and Foreign Trade at Unesc, in view of their competences and abilities compared to those required by Industry 4.0. It was characterized as a quantitative, descriptive, bibliographic and field research. The population consisted of 203 academics, with the effective participation of 80 who answered a questionnaire. We opted for the simple frequency linked to the literature. Academics agree that the fourth revolution will create more accurate and customizable production, and will also create a new demand for professionals, but they disagree with the fact that current models of education and training need updating. The impacts on the Administrator's profession are known to academics, and they know which professions will be most affected. Finally, it is clear that academics partly have in their profiles the skills and abilities necessary for Industry 4.0.
\end{abstract}

Keywords: Competencies. Skills. Industry 4.0. Revolution. 


\section{INTRODUÇÃO}

A Revolução Industrial (RI) transformou a vida das pessoas, com o acesso a grande variedade de produtos, novas formas de geração e distribuição de energia, meios de transporte mais eficientes e a migração em massa das pessoas para as cidades. $\mathrm{O}$ acesso à internet facilitou o contato tanto das pessoas quanto dos equipamentos ao redor do mundo e, esse contato tem se estreitado cada vez mais, como se o planeta estivesse encolhendo de tamanho (ASSOCIAÇÃO BRASILEIRA DE INTERNET INDUSTRIAL, 2017).

Marcada por transformações, a revolução industrial tem se consolidado ao passar dos anos, se tornando, um processo contínuo de atualização e transformação da indústria. A primeira RI usou água e o vapor para a mecanização da produção; a segunda RI usou a energia elétrica para criar produções em massa; a terceira RI utilizou a eletrônica e a tecnologia da informática para automatizar a produção e, a quarta RI - e atual, está crescendo sob os alicerces da digitalização da indústria, fundindo as tecnologias do mundo físico, digital e biológico (CÂNEDO, 2009).

Nos últimos anos, a $4^{\mathrm{a}} \mathrm{RI}$ vem ganhando força entre os países desenvolvidos. O primeiro país a iniciar esse processo foi a Alemanha no ano de 2011, a partir de um programa de incentivo a digitalização das indústrias, a intitulada Indústria 4.0. Essa indústria está fortemente atrelada com a Internet das Coisas (derivada do inglês IoT - Internet of Things), que se constitui em um sistema de objetos, cuja principal função é a troca de informações entre si em tempo real, possibilitando a esse sistema o seu autodiagnostico, autoconfiguração e auto otimização (CONFEDERAÇÃO NACIONAL DA INDÚSTRIA - CNI, 2016; DREHER, 2016).

A inserção da cultura digital nas empresas necessita que, todos os colaboradores estejam estritamente alinhados no processo, ajustando sua maneira de pensar e de agir às novas tecnologias. A partir deste cenário, surge a necessidade de uma visão interdisciplinar do colaborador, principalmente o profissional de Administração, que na sua grade curricular - na maior parte das universidades, não tem disciplinas voltadas para a perspectiva da Indústria 4.0.

Atualmente, o desenvolvimento das indústrias nos moldes da Indústria 4.0, há a implementação de maquinários modernos e, o qual devem possibilitar a troca de informações com os sistemas digitais existentes nas linhas de produção e com o restante da empresa. Para que os profissionais estejam capacitados e consigam lidar com os problemas provenientes desses maquinários, os mesmos devem possuir competências e habilidades específicas tornando-os aptos para atuarem dentro dessas empresas. 
A adaptação do profissional de Administração se faz importante para que haja sua consolidação tanto no mercado de trabalho quanto nas indústrias. Sua capacitação e formação devem ser coerentes com os requisitos exigidos pelas Indústrias 4.0. Neste contexto, o estudo tem por objetivo identificar a percepção dos acadêmicos dos cursos de Administração e Comércio Exterior da Universidade do Extremo Sul Catarinense - Unesc, diante das suas competências e habilidades frente àquelas requeridas pela Indústria 4.0.

\section{O PROFISSIONAL DA INDÚSTRIA 4.0}

Considerando que cada RI teve como base um desenvolvimento tecnológico específico, é imperativo afirmar que, ela trará mudanças, sejam elas positivas ou negativas. As razões pela qual a nova revolução tecnológica provocará mais agitações do que as revoluções anteriores são a velocidade em que ela está acontecendo; amplitude e profundidade, ou seja, está acontecendo simultaneamente em vários lugares e a transformação completa de sistemas inteiros, modificando cadeias inteiras de trabalho (SCHWAB, 2016). Ainda, afirma o autor que a Indústria 4.0 mudará a natureza do trabalho em todos os setores e ocupações. Diferentes categorias de trabalho serão atingidas, principalmente aquelas que realizam o trabalho mecânico repetitivo ou o trabalho manual de precisão. $O$ trabalho humano requerido por esta revolução cairá sobre as habilidades de resolução de problemas complexos e competências sociais (SCHWAB, 2016).

Para o World Economic Forum - WEF (2016), durante as revoluções industriais anteriores, muitas vezes as empresas levavam décadas para construir um sistema de treinamento para desenvolver grandes conjuntos de habilidades. Dado o ritmo e a escala de ruptura provocada pela $4^{\mathrm{a}}$ RI, no entanto, isso pode simplesmente não ser uma opção.

Os impactos da Indústria 4.0 sobre o emprego ainda requerem mais estudos, mas a automação da produção e a substituição dos trabalhadores já apresentam algumas profissões que poderão sofrer mudanças. Os países mais atingidos por estas serão aqueles que estão mais aptos a transformarem as suas indústrias aos moldes da Indústria 4.0, e isso está ligado ao nível de desenvolvimento, demográfico e socioeconômico do país (SCHWAB, 2016; WORLD ECONOMIC FORUM - WEF, 2016).

Ao mesmo passo que algumas profissões são afetadas, outras profissões serão criadas e beneficiadas pela Indústria 4.0. Um estudo encomendado pelo governo alemão, mostra que a digitalização da indústria poderá ter um impacto positivo na criação de postos de trabalho. Como resultado da pesquisa, 260 mil postos de trabalhos serão criados até 2030, contra 23 mil 
postos de trabalhos reduzidos, produzindo um saldo positivo de cerca de 240 mil postos de trabalho devido a digitalização da indústria (CNI, 2017).

Estes novos trabalhos serão baseados em uma matriz totalmente diferente de competências e habilidades das exigidas nas revoluções anteriores. O Quadro 1 explicita as principais competências e habilidades exigidas aos profissionais desta nova indústria.

Quadro 1 - Competências requeridas do profissional da Indústria 4.0.

\begin{tabular}{|c|c|c|}
\hline AUTOR & ANO & CARACTERÍSTICAS \\
\hline CNI & $2016 a$ & $\begin{array}{l}\text { Ter conhecimento multidisciplinar. } \\
\text { Ter elevado nível de conhecimento técnico. } \\
\text { Ter capacidade de interação com outras áreas do conhecimento. }\end{array}$ \\
\hline \multirow[b]{3}{*}{ WEF } & \multirow[b]{3}{*}{2016} & $\begin{array}{l}\text { Habilidades: } \\
\text { Cognitivas: flexibilidade cognitiva, criatividade, raciocínio lógico, sensibilidade } \\
\text { para problemas, raciocínio matemático e visualização. } \\
\text { Físicas: força física e destreza manual e precisão. }\end{array}$ \\
\hline & & $\begin{array}{l}\text { Competências básicas: } \\
\text { De conteúdo: aprendizagem ativa, expressão oral, compreensão de leitura, expressão } \\
\text { escrita e formação em TIC. } \\
\text { De processo: escuta ativa, pensamento crítico, monitoramento próprio e dos outros. }\end{array}$ \\
\hline & & $\begin{array}{l}\text { Competências transversais: } \\
\text { Sociais: coordenação de equipe, inteligência emocional, negociação, persuasão, } \\
\text { orientação de serviço, treinar e ensinar pessoas. } \\
\text { Sistêmicas: julgamento e tomada de decisão e análise sistêmica. } \\
\text { Resolução de Problemas Complexos: capacidade de resolver problemas } \\
\text { complexos. } \\
\text { Gestão de Recursos: gestão de recursos financeiros, gestão de recursos materiais, } \\
\text { gestão de pessoas e gestão de tempo. } \\
\text { Técnicas: manutenção e reparo de equipamentos, operação e controle de } \\
\text { equipamentos, programação, controle de qualidade, design de tecnologia e } \\
\text { experiência de usuário e solução de problemas. }\end{array}$ \\
\hline FESTO & $201-$ & $\begin{array}{l}\text { Ter formação multidisciplinar. } \\
\text { Ter conhecimento técnico para lidar com máquinas inteligentes. } \\
\text { Ter habilidade para aprender e se adaptar. } \\
\text { Observar e tomar decisões. } \\
\text { Criatividade. }\end{array}$ \\
\hline
\end{tabular}

Fonte: Elaboração própria a partir dos autores acima referenciados.

O Diretor-geral do SENAI, Rafael Luccesi, em uma entrevista à Confederação Nacional da Indústria - CNI (2018), declara que “as pessoas vão ter um processo contínuo de aprendizado ao longo da vida. Vão precisar se requalificar permanentemente para adquirir novas competências". Também argumenta que deve haver uma substituição forte de atividades de competências repetitivas, e possibilitarão a criação de novas oportunidades de trabalho para interpretação de dados - em grande quantidade, devido ao big data, criticidade de dados e tomada de decisões. 


\section{ARTIGO}

INOVAÇÃo

\section{PROCEDIMENTOS METODOLÓGICOS}

A pesquisa tem abordagem quantitativa. Este método caracteriza-se pelo emprego da quantificação, tanto na coleta de informações quanto no tratamento das mesmas (RICHARDSON, 1985). Neste estudo, a abordagem quantitativa foi aplicada uma vez que, buscou-se a precisão dos dados coletados, tanto na sua elaboração quanto na sua análise, fazendo uso, portanto, de um tratamento quantitativo.

Quanto aos fins de investigação, a pesquisa se classificou como descritiva, pois de acordo com Michel (2015) a pesquisa descritiva verifica, descreve e explica problemas, fatos ou fenômenos da vida real, com a precisão possível, observando e fazendo relações, conexões, considerando a influência que o ambiente exerce sobre eles. Neste sentido, a pesquisa descritiva se faz necessária para que se possa identificar as principais competências e habilidades já presentes nos acadêmicos dos cursos de Administração e Comércio Exterior da Unesc perante às exigencias da Indústria 4.0.

Os meios de investigação se caracterizam em um primeiro momento a partir de uma pesquisa bibliográfica, o qual é realizado consulta em livros, artigos, periódicos, anais, bancos e bases de dados, relatórios, teses, dissertações etc, com o objetivo de traçar as competências do profissional (MICHEL, 2015). A pesquisa de campo, no ambiente natural, é particularmente importante na pesquisa social, apropriada para estudos de indivíduos, grupos, comunidades, organizações, sociedades, considerando que, para a pesquisa social, mais importante que encontrar soluções é explicar os fenômenos, entender realidades, criar significados sociais, além de confrontar a teoria na prática (MICHEL, 2015). Esta, por sua vez, se justifica pelo fato de que era necessário saber o conhecimento dos acadêmicos sobre os princípios e características da Indústria 4.0, como também se as suas competências e habilidades estão alinhadas com àquelas requeridas por esta nova RI. Desta forma, a aplicação da mesma foi efetuada diretamente com os acadêmicos. O questionário usado para a aplicação da pesquisa deste estudo foi baseado em Souza (2018).

Ainda, a população foi pautada em acadêmicos das $7^{\mathrm{a}}$ e $8^{\mathrm{a}}$ fases dos cursos de Administração e Comércio Exterior da Unesc, compreendendo os acadêmicos graduandos no semestre em que foi realizada a pesquisa, como demonstrado na Tabela 1. 
Tabela 1 - Composição das $7^{\mathrm{a}}$ e $8^{\mathrm{a}}$ fases dos cursos de Administração e Comércio Exterior da UNESC.

\begin{tabular}{l|r}
\multicolumn{1}{c|}{ CURSOS } & POPULAÇÃO/ACADÊMICOS \\
\hline Administração - Matutino & 20 \\
Administração - Noturno & 121 \\
Administração - Comércio Exterior & 62 \\
\hline TOTAL & $\mathbf{2 0 3}$ \\
\hline
\end{tabular}

Fonte: Elaboração própria a partir de dados da pesquisa.

Para a determinação da população foi utilizada a lista de alunos dos referidos cursos, disponibilizadas, o qual se obteve um total de 203 acadêmicos concluintes de curso.

No que diz respeito a amostragem, Mattar (2001) a define como uma pequena parte da população original que irá compor a amostra, ficando a caráter do pesquisador decidir quais serão os elementos a serem incluídos. Assim, dos 203 acadêmicos selecionados como população-alvo, foi aplicado o questionário com 80 acadêmicos, considerando a acessibilidade, disponibilidade e presença dos mesmos no momento da aplicação em sala de aula. Este foi realizado em quatro momentos: o primeiro momento participaram 32 acadêmicos; no segundo momento sete acadêmicos; no terceiro momento 26 acadêmicos, e no último e quarto momentos, foram 15 acadêmicos. A Tabela 2 demonstra a quantidade de acadêmicos respondentes da pesquisa.

Tabela 2 - Amostra dos acadêmicos respondentes da pesquisa.

\begin{tabular}{l|r}
\multicolumn{1}{c|}{ CURSOS } & \multicolumn{1}{c}{ AMOSTRA /ACADÊMICOS } \\
\hline Administração - Matutino & 0 \\
Administração - Noturno & 58 \\
Administração - Comércio Exterior & 22 \\
\hline TOTAL & $\mathbf{8 0}$ \\
\hline
\end{tabular}

Fonte: Elaboração própria a partir de dados da pesquisa.

Para a realização da pesquisa de campo, fez-se o uso essencialmente de fontes primárias. As fontes primárias, segundo Diehl e Tatim (2004), consistem na obtenção de dados em primeira mão pelo pesquisador, e que estarão fazendo parte da composição da pesquisa de campo. Para a coleta de dados primários, é necessário definir qual instrumento será utilizado para tal. Neste sentido, o questionário, instrumento de pesquisa pertencente a técnica quantitativa, fora escolhido para estruturar tal estudo (MICHEL, 2015). O questionário foi dividido em três blocos constituídos por perguntas fechadas e de múltipla escolha, com o intuito de responder e abranger os seguintes assuntos: $i$ ) perfil dos acadêmicos entrevistados; ii) 
INOVAÇÃO

conhecimento prévio dos acadêmicos dos cursos de Administração e Comércio Exterior da Unesc sobre as características da Indústria 4.0; iii) comparar as competências e habilidades dos acadêmicos dos cursos de Administração e Comércio Exterior da Unesc frente às requeridas pela nova RI; e $i v$ ) verificar o conhecimento dos impactos desta nova revolução na profissão do Administrador.

A aplicação do instrumento de pesquisa foi realizada no período de 08 de outubro a 11 de outubro de 2018, após entrega dos questionários em meio físico aos acadêmicos dos cursos de Administração e Comércio Exterior da Unesc. O instrumento de pesquisa teve como base o questionário já utilizado por Souza (2018). A partir deste instrumento, foram realizadas adaptações para adequar aos objetivos do estudo.

Para a análise dos dados utilizou-se a técnica de análise de frequência simples, pois de acordo com Duarte e Furtado (2014, p. 142): "mais importante do que apontar a representatividade dos resultados quantitativos é refletir e interpretar o que significam, extrapolando para outras fontes e informações constantes no trabalho e fazendo inferências".

\section{APRESENTAÇÃO E DISCUSSÃO DOS RESULTADOS}

\subsection{PERFIL DOS ACADÊMICOS}

Observando a Tabela 3, nota-se que 52,5\% dos acadêmicos do curso são do gênero masculino, e 47,5\% do gênero feminino; além disso, percebe-se também que 72,5\% dos alunos são do curso de Administração, e 27,5\% são do curso de Comércio Exterior.

Tabela 3 - Perfil dos acadêmicos.

\begin{tabular}{l|l|c|r}
\hline \multirow{2}{*}{ CURSO } & \multirow{2}{*}{ GÊNERO } & \multicolumn{2}{c}{ TOTAL } \\
\cline { 3 - 4 } & & QTD. & \% \\
\hline \multirow{2}{*}{$\begin{array}{l}\text { Administração - Linha de Formação Específica em } \\
\text { Administração de Empresas }\end{array}$} & Feminino & 27 & $33,75 \%$ \\
\cline { 2 - 4 } & Masculino & 31 & $38,75 \%$ \\
\hline \multirow{2}{*}{$\begin{array}{l}\text { Administração - Linha de Formação Específica em } \\
\text { Comércio Exterior }\end{array}$} & Feminino & 11 & $13,75 \%$ \\
\cline { 2 - 4 } & Masculino & 11 & $13,75 \%$ \\
\hline TOTAL & & $\mathbf{8 0}$ & $\mathbf{1 0 0 \%}$ \\
\hline
\end{tabular}

Fonte: Elaboração própria a partir de dados da pesquisa.

No que diz respeito as cidades em que cada acadêmico reside, a Tabela 4 demonstra a quantidade de alunos residentes das regiões como AMESC - Associação dos Municípios do Extremo Sul Catarinense, e AMREC - Associação dos Municípios da Região Carbonífera, além 
de cidades do Estado do Rio Grande do Sul. Na região da AMESC estão concentrados $15 \%$ dos acadêmicos; na região da AMREC são 81,25\% dos alunos, e restante, 3,75\% estão em cidades do Rio Grande do Sul.

Tabela 4 - Cidade onde residem os acadêmicos.

\begin{tabular}{|c|c|c|c|}
\hline \multirow{2}{*}{ REGIÃO } & \multirow{2}{*}{ CIDADE } & \multicolumn{2}{|c|}{ TOTAL } \\
\hline & & FREQUÊNCIA & $\%$ \\
\hline \multirow{8}{*}{ AMESC } & Araranguá & 3 & $3,75 \%$ \\
\hline & Jacinto Machado & 1 & $1,25 \%$ \\
\hline & Maracajá & 1 & $1,25 \%$ \\
\hline & Meleiro & 1 & $1,25 \%$ \\
\hline & Praia Grande & 1 & $1,25 \%$ \\
\hline & Santa Rosa do Sul & 1 & $1,25 \%$ \\
\hline & Sombrio & 3 & $3,75 \%$ \\
\hline & Turvo & 1 & $1,25 \%$ \\
\hline \multirow{9}{*}{ AMREC } & Cocal do Sul & 3 & $3,75 \%$ \\
\hline & Criciúma & 37 & $46,25 \%$ \\
\hline & Forquilhinha & 6 & $7,50 \%$ \\
\hline & Içara & 6 & $7,50 \%$ \\
\hline & Lauro Müller & 1 & $1,25 \%$ \\
\hline & Morro da Fumaça & 5 & $6,25 \%$ \\
\hline & Nova Veneza & 2 & $2,50 \%$ \\
\hline & Treviso & 2 & $2,50 \%$ \\
\hline & Urussanga & 3 & $3,75 \%$ \\
\hline ESTADO DO RIO & Morrinhos do Sul & 1 & $1,25 \%$ \\
\hline GRANDE DO SUL & Torres & 2 & $2,50 \%$ \\
\hline \multicolumn{2}{|l|}{ TOTAL } & 80 & $100 \%$ \\
\hline
\end{tabular}

Fonte: Elaboração própria a partir de dados da pesquisa.

Evidencia-se, assim, a importância dos cursos de Administração e Comércio Exterior da Unesc, para a região, pois esta pesquisa mostra a abrangência da Unesc em várias cidades.

\subsection{CONHECIMENTO PRÉVIO SOBRE A INDÚSTRIA 4.0}

Em todos os contextos dos acadêmicos, a tecnologia se faz importante. No contexto pessoal, 52,50\% dos acadêmicos responderam que a tecnologia é muito importante, e o mesmo segue para o contexto acadêmico com $68,75 \%$. De todos os ambientes em que a tecnologia faz parte, o resultado mais expressivo foi no ambiente profissional, com $0,00 \%$ de respostas para 


\section{ARTIGO}

\section{INOVAÇÃO}

não importante e pouco importante, mas para importante e muito importante, as respostas foram de $18,75 \%$ e $81,25 \%$, respectivamente, apresentado na Tabela 5.

Tabela 5 - Uso da tecnologia no dia a dia.

\begin{tabular}{|c|c|c|c|c|c|c|}
\hline \multirow{3}{*}{$\begin{array}{l}\text { CARACTERÍSTICAS/ } \\
\text { ELEMENTO }\end{array}$} & \multicolumn{6}{|c|}{$\begin{array}{l}\text { IMPORTÂNCIA DA TECNOLOGIA NO AMBIENTE PESSOAL, ACADÊMICO E } \\
\text { PROFISSIONAL }\end{array}$} \\
\hline & \multicolumn{2}{|c|}{ A) PESSOAL } & \multicolumn{2}{|c|}{ B) ACADÊMICO } & \multicolumn{2}{|c|}{ C) PROFISSIONAL } \\
\hline & $\mathbf{F}$ & $\%$ & $\mathbf{F}$ & $\%$ & $\mathbf{F}$ & $\%$ \\
\hline Não é importante & 1 & $1,25 \%$ & 0 & $0,00 \%$ & 0 & $0,00 \%$ \\
\hline Pouco importante & 1 & $1,25 \%$ & 0 & $0,00 \%$ & 0 & $0,00 \%$ \\
\hline Importante & 36 & $45,00 \%$ & 25 & $31,25 \%$ & 15 & $18,75 \%$ \\
\hline Muito importante & 42 & $52,50 \%$ & 55 & $68,75 \%$ & 65 & $81,25 \%$ \\
\hline TOTAL & 80 & $100 \%$ & 80 & $100 \%$ & 80 & $100 \%$ \\
\hline
\end{tabular}

Fonte: Elaboração própria a partir de dados da pesquisa.

A tecnologia gerou grandes rupturas na sociedade, criando o mundo para o seu estágio atual de desenvolvimento e globalização. Hoje, a humanidade passa por uma mudança inegável, a chegada da $4^{\mathrm{a}}$ RI. Ela trará benefícios para as indústrias com a automatização de processos, diminuindo os custos de produção; para os clientes oportunizará a customização dos produtos, e para os trabalhadores, a substituição de mão de obra pesada, como também a criação de muitos novos empregos (SCHWAB, 2016).

De acordo com a Tabela 6, os acadêmicos possuem 53,75\% de conhecimento sobre os acontecimentos da Indústria 4.0. Os dados não se mostram alarmantes, pois apenas 1,25\% dos acadêmicos não possuem nenhum tipo de conhecimento acerca da $4^{\mathrm{a}} \mathrm{RI}$.

Tabela 6 - Conhecimento prévio sobre a Indústria 4.0.

\begin{tabular}{l|c|r}
\hline \multirow{2}{*}{$\begin{array}{c}\text { CARACTERÍSTICAS/ } \\
\text { ELEMENTO }\end{array}$} & \multicolumn{2}{c}{$\begin{array}{c}\text { O NÍVL DE CONHECIMENTO SOBRE OS ACONTECIMENTOS DA } \\
\text { QUARTA REVOLUÇÃO INDUSTRIAL (INDÚSTRIA 4.0) }\end{array}$} \\
\cline { 2 - 3 } & F & \% \\
\hline Nenhum conhecimento & 1 & $1,25 \%$ \\
Pouco conhecimento & 30 & $37,50 \%$ \\
Conhecimento intermediário & 43 & $53,75 \%$ \\
Muito conhecimento & 6 & $7,50 \%$ \\
\hline TOTAL & $\mathbf{8 0}$ & $\mathbf{1 0 0 \%}$ \\
\hline
\end{tabular}

Fonte: Elaboração própria a partir de dados da pesquisa.

A $4^{\mathrm{a}}$ RI traz características únicas que são os principais geradores de mudança. Tais características unidas, se tornam um conjunto completo de máquinas interligadas, onde tudo o que nele estiver conectado, será capaz de trocar informações, solucionar problemas e o autocontrole de forma independente (SCHWAB, 2016).

Sobre o nível de conhecimento dos acadêmicos acerca das principais tecnologias que caracterizam a Indústria 4.0 (Tabela 7), para nenhum conhecimento, o resultado mais 
expressivo foi para a Big Data, que contou com um nível 22,50\% de nenhum conhecimento; para pouco conhecimento os mais expressivos foram fábricas inteligentes e Big Data, com $52,50 \%$ e 51,25\%, respectivamente; para conhecimento intermediário, o mais expressivo foi Internet das coisas, com $45,00 \%$, e por último, para muito conhecimento, os resultados mais expressivos foram Internet das coisas e Inteligência Artificial, com 7,50\%.

Tabela 7 - Conhecimento prévio acerca das tecnologias da Indústria 4.0.

\begin{tabular}{l|c|c|c|c|c|c|c|c}
\hline \multirow{2}{*}{$\begin{array}{c}\text { CARACTERÍSTICAS/ } \\
\text { ELEMENTO }\end{array}$} & \multicolumn{6}{|c|}{ NÍVEL DE CONHECIMENTO SOBRE AS TECNOLOGIAS QUE CARACTERIZAM } \\
A INDÚSTRIA 4.0
\end{tabular}

Fonte: Elaboração própria a partir de dados da pesquisa.

No que diz respeito a Tabela 8 , referente a utilização da nuvem para armazenamento de arquivos pessoais, acadêmicos e profissionais, o que eles menos utilizam é o armazenamento de arquivos profissionais, com 35,00\% das respostas; com pouca utilização, temos os arquivos pessoais e acadêmicos, com 35,00\% e 32,50\%, respectivamente; com utilização frequente, temos os arquivos acadêmicos com $31,25 \%$, e com utilização constante, temos os arquivos profissionais com $23,75 \%$.

Tabela 8 - Nível de utilização da nuvem para armazenamento de arquivos.

\begin{tabular}{|c|c|c|c|c|c|c|}
\hline \multirow{3}{*}{$\begin{array}{l}\text { CARACTERÍSTICAS/ } \\
\text { ELEMENTO }\end{array}$} & \multicolumn{6}{|c|}{ NÍVEL DE UTILIZAÇÃO DA NUVEM PARA ARMAZENAMENTO DE ARQUIVOS } \\
\hline & \multicolumn{2}{|c|}{$\begin{array}{l}\text { A) ARQUIVOS } \\
\text { PESSOAIS }\end{array}$} & \multicolumn{2}{|c|}{$\begin{array}{l}\text { B) ARQUIVOS } \\
\text { ACADÊMICOS }\end{array}$} & \multicolumn{2}{|c|}{$\begin{array}{l}\text { C) ARQUIVOS } \\
\text { PROFISSIONAIS }\end{array}$} \\
\hline & $\mathbf{F}$ & $\%$ & $\mathbf{F}$ & $\%$ & $\mathbf{F}$ & $\%$ \\
\hline Não utiliza & 22 & $27,50 \%$ & 15 & $18,75 \%$ & 28 & $35,00 \%$ \\
\hline Utiliza pouco (as vezes) & 28 & $35,00 \%$ & 26 & $32,50 \%$ & 15 & $18,75 \%$ \\
\hline Utiliza frequentemente & 15 & $18,75 \%$ & 25 & $31,25 \%$ & 18 & $22,50 \%$ \\
\hline Sempre utiliza & 15 & $18,75 \%$ & 14 & $17,50 \%$ & 19 & $23,75 \%$ \\
\hline TOTAL & 80 & $100 \%$ & 80 & $100 \%$ & 80 & $100 \%$ \\
\hline
\end{tabular}

Fonte: Elaboração própria a partir de dados da pesquisa.

Com as fábricas inteligentes, a inteligência artificial, a internet das coisas e a big data, será possível criar indústrias de baixo custo, onde as máquinas poderão reproduzir diferentes 


\section{ARTIGO}

INOVAÇÃO

modelos de produtos em sequência, sem qualquer necessidade de parada para configuração, permitindo personalizações em massa com alta velocidade. Isso fará com que empresas possuam um mix mais variado de produtos, e com que clientes possam obter produtos que sejam eficientes para aquilo que desejam (SCHWAB, 2016).

Referente a criação de uma linha de produção mais precisa e personalizável pela Indústria 4.0 (Tabela 9), 62,50\% dos acadêmicos concordam parcialmente com a afirmação, enquanto $15,00 \%$ discordam, e 22,50\% concordam totalmente. A maioria dos acadêmicos concorda como fato de que a quarta revolução industrial criará indústrias melhores, mas não com capacidade de personalização e precisão.

Tabela 9 - A Indústria 4.0 criará linha de produção mais precisa e personalizável.

\begin{tabular}{l|c|r}
\hline \multirow{2}{*}{$\begin{array}{c}\text { CARACTERÍSTICAS/ } \\
\text { ELEMENTO }\end{array}$} & \multicolumn{2}{|c}{$\begin{array}{c}\text { A INDÚSTRIA 4.0 CRIARÁ LINHA DE PRODUÇÃO MAIS } \\
\text { PRECISA E PERSONALIZÁVEL }\end{array}$} \\
\cline { 2 - 3 } & F & \% \\
\hline Discordo parcialmente & 12 & $15,00 \%$ \\
Concordo parcialmente & 50 & $62,50 \%$ \\
Concordo totalmente & 18 & $22,50 \%$ \\
\hline TOTAL & $\mathbf{8 0}$ & $\mathbf{1 0 0 \%}$ \\
\hline
\end{tabular}

Fonte: Elaboração própria a partir de dados da pesquisa.

Atualmente, se enfrenta uma grande diversidade de desafios fascinantes; entre eles, o mais intenso e importante é o entendimento e a modelagem da nova revolução tecnológica, a qual implica em nada menos que a transformação de toda a humanidade. É o início de uma revolução que alterará profundamente a forma em que a sociedade vive, trabalha e se relaciona (SCHWAB, 2016).

É perceptível que, há pouco conhecimento sobre a Indústria 4.0 disseminado entre os acadêmicos dos cursos de Administração e Comércio Exterior entrevistados. Este cenário precisa ser revertido, pois os mesmos serão os profissionais do futuro e, como precursores da nova revolução, precisam estar capacitados e aptos à nova realidade.

A próxima subseção abarcará temas como os impactos provocados pela Indústria 4.0 na profissão do Administrador, bem como as competências e habilidades por ela requerida em paralelo com as competências e habilidades presentes nos acadêmicos, e também quais meios foram utilizados para que os acadêmicos adquirissem conhecimento sobre a Indústria 4.0. 


\subsection{IMPACTOS DA INDÚSTRIA 4.0 NAS COMPETÊNCIAS E NA PROFISSÃO DO ADMINISTRADOR}

No que diz respeito a Tabela 10, referente as competências e habilidades requeridas pela Indústria 4.0 frente as competências e habilidades dos acadêmicos, as características menos presentes são raciocínio lógico e resolução de problemas complexos, com 12,50\% e 8,75\%, respectivamente. Quanto às características do perfil dos acadêmicos, as que mais se encontram presentes são gestão de tempo e resolução de problemas complexos, com 66,25\% e 62,50\%, respectivamente; para as características que fazem parte do perfil dos acadêmicos, os destaques vão para trabalho corporativo ou em equipe e disposição para tomar e compartilhar decisões, com $43,75 \%$ para ambas as características.

Tabela 10 - Competências e habilidades da Indústria 4.0 versus competências e habilidades dos acadêmicos.

\begin{tabular}{|c|c|c|c|c|c|c|c|c|c|c|}
\hline \multirow{3}{*}{$\begin{array}{l}\text { CARACTERÍSTICAS/ } \\
\text { ELEMENTO }\end{array}$} & \multicolumn{10}{|c|}{ COMPETÊNCIAS E HABILIDADES PRESENTES NO PERFIL } \\
\hline & \multicolumn{2}{|c|}{$\begin{array}{l}\text { A) TRABALHO } \\
\text { CORPORATIVO } \\
\text { E EM EQUIPE }\end{array}$} & \multicolumn{2}{|c|}{$\begin{array}{l}\text { B) GESTÃO DE } \\
\text { TEMPO }\end{array}$} & \multicolumn{2}{|c|}{$\begin{array}{l}\text { C) RESOLUÇÃO } \\
\text { DE PROBLEMAS } \\
\text { COMPLEXOS }\end{array}$} & \multicolumn{2}{|c|}{$\begin{array}{c}\text { D) } \\
\text { RACIOCÍNIO } \\
\text { ANALÍTICO }\end{array}$} & \multicolumn{2}{|c|}{$\begin{array}{l}\text { E) DISPOSIÇÃO } \\
\text { PARA TOMAR E } \\
\text { COMPARTILHAR } \\
\text { DECISÕES }\end{array}$} \\
\hline & $\mathbf{F}$ & $\%$ & $\mathbf{F}$ & $\%$ & $\mathbf{F}$ & $\%$ & $\mathbf{F}$ & $\%$ & $\mathbf{F}$ & $\%$ \\
\hline $\begin{array}{l}\text { Não poss } \\
\text { caracterí }\end{array}$ & 5 & $6,25 \%$ & 3 & $3,75 \%$ & 7 & $8,75 \%$ & 10 & $12,50 \%$ & 4 & $5,00 \%$ \\
\hline $\begin{array}{l}\text { Sim, possuo esta } \\
\text { característica em parte }\end{array}$ & 40 & $50,00 \%$ & 53 & $66,25 \%$ & 50 & $62,50 \%$ & 45 & $56,25 \%$ & 41 & $51,25 \%$ \\
\hline $\begin{array}{l}\text { Esta característica faz } \\
\text { parte do meu perfil }\end{array}$ & 35 & $43,75 \%$ & 24 & $30,00 \%$ & 23 & $28,75 \%$ & 25 & $31,25 \%$ & 35 & $43,75 \%$ \\
\hline TOTAL & 80 & $100 \%$ & 80 & $100 \%$ & 80 & $100 \%$ & 80 & $100 \%$ & 80 & $100 \%$ \\
\hline
\end{tabular}

Fonte: Elaboração própria a partir de dados da pesquisa.

Uma vez que esta revolução poderá destruir muitos postos de trabalho, ela também irá criar outros ainda inexistentes. Este fato acontecerá devido à grande transformação que a Indústria 4.0 tem causado no mercado. De acordo com estudo realizado pelas indústrias alemãs, serão ofertadas 240 mil novas vagas de emprego, em relação a 30 mil postos de trabalho que serão fechados (CNI, 2017).

Com relação a criação de novas demandas por profissionais (Tabela 11), 1,25\% dos acadêmicos discordam totalmente; 5,00\% discordam parcialmente; 50,00\% concordam parcialmente, e 43,75\% concordam totalmente. Os acadêmicos acreditam que, com a chegada desta nova revolução, novos profissionais serão requeridos pelo mercado de trabalho para exercerem profissões que ainda não são conhecidas. 
Tabela 11 - Criação de novas demandas por profissionais.

\begin{tabular}{l|c|r}
\hline \multicolumn{1}{c|}{ CARACTERÍSTICAS/ } & \multicolumn{2}{|c}{ HAVERÁ CRIAÇÃO DE NOVAS DEMANDAS POR PROFISSIONAIS } \\
\cline { 2 - 3 } \multicolumn{1}{c}{ ELEENTO } & $\mathbf{F}$ & $\mathbf{\%}$ \\
\cline { 2 - 3 } Discordo totalmente & 1 & $1,25 \%$ \\
Discordo parcialmente & 4 & $5,00 \%$ \\
Concordo parcialmente & 40 & $50,00 \%$ \\
Concordo totalmente & 35 & $43,75 \%$ \\
\hline TOTAL & $\mathbf{8 0}$ & $\mathbf{1 0 0 \%}$ \\
\hline
\end{tabular}

Fonte: Elaboração própria a partir de dados da pesquisa.

A nova demanda por profissionais necessitará de novos modelos de capacitação da mãode-obra, pois esta, no momento, está carente de conhecimento. Os futuros profissionais precisam adequar-se às mudanças inerentes à Indústria 4.0 e, para que isso aconteça, é necessária a reformulação dos atuais modelos de formação e capacitação profissional para que estejam alinhadas às novas demandas do mercado de trabalho. A atualização nas grades curriculares dos cursos de Administração é crucial para que os acadêmicos estejam preparados. Para isso, empresas e universidades devem trabalhar em parceria, alinhando a teoria à prática (AIRES; KEMPNER-MOREIRA; FREIRE, 2017).

No que diz respeito a Tabela 12 , referente a mudança nos modelos atuais de capacitação profissional, 7,50\% discordam totalmente; $52,50 \%$ concordam parcialmente, e $40,00 \%$ dos acadêmicos concordam totalmente com esta afirmação. Deste modo, percebe-se o grande discernimento entre os acadêmicos de que, o modelo de ensino atual não é suficiente para suprir os conhecimentos que são necessários para o desenvolvimento de trabalhos com qualidade provenientes da Indústria 4.0.

Tabela 12 - Mudanças na capacitação profissional.

\begin{tabular}{l|c|r}
\hline \multirow{2}{*}{\begin{tabular}{c} 
CARACTERÍSTICAS/ \\
\multicolumn{1}{c|}{ ELEMENTO }
\end{tabular}} & HAVERÁ MUDANÇA NOS MODELOS DE CAPACITAÇÃO PROFISSIONAL \\
\cline { 2 - 3 } & 6 & $\%$ \\
\hline Discordo totalmente & 42 & $7,50 \%$ \\
Discordo parcialmente & 32 & $52,50 \%$ \\
Concordo parcialmente & $\mathbf{8 0}$ & $40,00 \%$ \\
\hline TOTAL & $\mathbf{1 0 0 \%}$ \\
\hline
\end{tabular}

Fonte: Elaboração própria a partir de dados da pesquisa.

Para os administradores, o mercado de trabalho é muito amplo, podendo exercer função em várias áreas, tais como financeiro, recursos humanos, logística, marketing, produção, comércio exterior, gestão ambiental, gestão de processos ou planejamento estratégico, possuindo vários postos de trabalhos distribuídos dentro de uma empresa, desde o setor de produção até ao setor da alta gestão (UNIVERSIDADE DO EXTREMO SUL CATARINENSE, 2018). 
Referente aos impactos da Indústria 4.0 sobre a profissão do Administrador (Tabela 13), no quesito de não automatização, a profissão de gerente de recursos humanos e diretores, contaram com os menores índices de automatização, com 22,50\% e 21,25\%, respectivamente; já para a pouca probabilidade de automatização, gerente de recursos humanos e gerente de vendas contaram com os índices de 41,25\% e 36,25\%, respectivamente; com a probabilidade intermediária, gerente de vendas e empreendedorismo e inovação contaram os maiores índices e automatização, com $36,25 \%$ e $28,75 \%$, respectivamente, e por último, os índices que contaram com maiores chances de automatização foram os responsáveis por cálculos financeiros e empreendedorismo e inovação, com $53,75 \%$ e $28,75 \%$, respectivamente.

Tabela 13 - Impactos na profissão do Administrador.

\begin{tabular}{|c|c|c|c|c|c|c|c|c|c|c|}
\hline \multirow{3}{*}{$\begin{array}{l}\text { CARACTERÍSTICAS/ } \\
\text { ELEMENTO }\end{array}$} & \multicolumn{10}{|c|}{ IMPACTOS DA INDÚSTRIA 4.0 SOBRE A PROFISSÃO DO ADMINISTRADOR } \\
\hline & \multicolumn{2}{|c|}{$\begin{array}{l}\text { A) GERENTE } \\
\text { DE RECURSOS } \\
\text { HUMANOS }\end{array}$} & \multicolumn{2}{|c|}{$\begin{array}{l}\text { B) GERENTE } \\
\text { DE VENDAS }\end{array}$} & \multicolumn{2}{|c|}{$\begin{array}{c}\text { C) } \\
\text { DIRETORES }\end{array}$} & \multicolumn{2}{|c|}{\begin{tabular}{|c|} 
D) RESPONSÁVEL \\
POR CÁLCULOS \\
FINANCEIROS E \\
FISCAIS \\
\end{tabular}} & \multicolumn{2}{|c|}{\begin{tabular}{|c} 
E) \\
EMPREENDEDORIS \\
MO E INOVAÇÃO
\end{tabular}} \\
\hline & $\mathbf{F}$ & $\%$ & $\mathbf{F}$ & $\%$ & $\mathbf{F}$ & $\%$ & $\mathbf{F}$ & $\%$ & $\mathbf{F}$ & $\%$ \\
\hline Não serão automatizados & 18 & $22,50 \%$ & 10 & $12,50 \%$ & 17 & $21,25 \%$ & 2 & $2,50 \%$ & 13 & $16,25 \%$ \\
\hline Pouca probabilidade & 33 & $41,25 \%$ & 29 & $36,25 \%$ & 27 & $33,75 \%$ & 14 & $17,50 \%$ & 21 & $26,25 \%$ \\
\hline $\begin{array}{l}\text { Probabilidade } \\
\text { intermediária }\end{array}$ & 23 & $28,75 \%$ & 29 & $36,25 \%$ & 22 & $27,50 \%$ & 21 & $26,25 \%$ & 23 & $28,75 \%$ \\
\hline Muita probabilidade & 6 & $7,50 \%$ & 12 & $15,00 \%$ & 14 & $17,50 \%$ & 43 & $53,75 \%$ & 23 & $28,75 \%$ \\
\hline TOTAL & 80 & $100 \%$ & 80 & $100 \%$ & 80 & $100 \%$ & 80 & $100 \%$ & 80 & $100 \%$ \\
\hline
\end{tabular}

Fonte: Elaboração própria a partir de dados da pesquisa.

A internet hoje é uma ferramenta disponível e acessível para quase toda a população mundial, estando praticamente a todo momento, na palma das mãos. Em décadas anteriores, a criação do conhecimento era feita apenas em escritos de papel e livros, o que dificultava a sua disseminação. Hoje, o conhecimento está disseminado por todo o mundo, disponível em qualquer tipo de plataforma, seja um smartphone, tablet, notebook ou computador. A criação de conteúdos qualificantes não se dá mais somente em sala de aula, cursos a distância facilitam e colaboram para que isso aconteça, seja pela comodidade e praticidade, ou pelo seu baixo preço de aquisição (ASSOCIAÇÃO BRASILEIRA DE INTERNET INDUSTRIAL, 2017).

Quando questionados sobre os meios de aquisição de conhecimento ligados à Indústria 4.0, 55,00\% dos acadêmicos adquiriram tais conhecimentos através da leitura de artigos, jornais, revistas ou livros; $52,50 \%$ através de reportagens ou palestras; 35,00\% por meio de disciplinas ministradas nos cursos de Administração e Comércio Exterior da Unesc, e 7,50\% por outros meios. 
Tabela 14 - Meios de aquisição de conhecimento.

\begin{tabular}{|c|c|c|c|c|c|c|c|c|}
\hline \multirow{3}{*}{$\begin{array}{l}\text { CARACTERÍSTICAS/ } \\
\text { ELEMENTO }\end{array}$} & \multicolumn{8}{|c|}{ MEIOS DE AQUISIÇÃO DE CONHECIMENTO } \\
\hline & \multicolumn{2}{|c|}{$\begin{array}{c}\text { A) LEITURA DE } \\
\text { ARTIGOS, JORNAIS, } \\
\text { REVISTAS OU LIVROS }\end{array}$} & \multicolumn{2}{|c|}{$\begin{array}{l}\text { B) REPORTAGENS } \\
\text { OU PALESTRAS }\end{array}$} & \multicolumn{2}{|c|}{$\begin{array}{l}\text { C) DISCIPLINAS } \\
\text { DO CURSO }\end{array}$} & \multicolumn{2}{|c|}{$\begin{array}{l}\text { D) OUTROS } \\
\text { MEIOS }\end{array}$} \\
\hline & $\mathbf{F}$ & $\%$ & $\mathbf{F}$ & $\%$ & $\mathbf{F}$ & $\%$ & $\mathbf{F}$ & $\%$ \\
\hline Sim & 44 & $55,00 \%$ & 42 & $52,50 \%$ & 28 & $35,00 \%$ & 6 & $7,50 \%$ \\
\hline Não & 36 & $45,00 \%$ & 38 & $47,50 \%$ & 52 & $65,00 \%$ & 74 & $92,50 \%$ \\
\hline TOTAL & 80 & $100 \%$ & 80 & $100 \%$ & 80 & $100 \%$ & 80 & $100 \%$ \\
\hline
\end{tabular}

Fonte: Elaboração própria a partir de dados da pesquisa.

Percebe-se que 65,00\% dos acadêmicos responderam que não receberam nenhum tipo de conhecimento da Indústria 4.0 através de disciplinas ministradas em seus respectivos cursos, isso representa um dado alarmante para as empresas, pois as mesmas estarão recrutando recémgraduados que saíram das instituições de ensino, despreparados para exercerem as funções que esta empresa necessita.

Destacando alguns dados importantes extraídos da pesquisa, algumas respostas foram dadas em “Outros meios", ou seja, meios não citados de aquisição de conhecimento, como por exemplo artigos divulgados em redes sociais como LinkedIn, Semana Acadêmica dos cursos de Administração e Comércio Exterior, e também por meio de pesquisas feitas pelos próprios acadêmicos na internet.

No que diz respeito ao acadêmico estar preparado para o mercado de trabalho da Indústria 4.0, 58,75\% dos acadêmicos responderam que estão aptos para atuarem neste mercado, o que representa um médio desempenho dos acadêmicos dos cursos de Administração e Comércio Exterior da Unesc, frente a nova Revolução Industrial.

Tabela 15 - Sentimento de profissional preparado.

\begin{tabular}{l|c|r}
\hline \multirow{2}{*}{$\begin{array}{c}\text { CARACTERÍSTICAS/ } \\
\text { ELEMENTO }\end{array}$} & \multicolumn{3}{|c}{ OS ACADÊMICOS SENTEM-SE PREPARADOS PARA O MERCADO DE } \\
TRABALHO DA INDÚSTRIA 4.0?
\end{tabular}

Fonte: Elaboração própria a partir de dados da pesquisa.

Se analisar o percentual de acadêmicos que receberam conhecimento pelas disciplinas dos cursos com o percentual de acadêmicos que não se sentem preparados para este novo mercado, temos um resultado alarmante sobre a falta de conhecimento sobre a Indústria $4.0 \mathrm{e}$ suas demandas para o futuro, de modo que, é necessário repensar os modelos de ensino atuais para que estes estejam alinhados com as novas propostas apresentadas pela $4^{\mathrm{a}} \mathrm{RI}$. 
Frente a isto, para a verificação da existência dessas características no perfil dos acadêmicos, o Quadro 18 apresenta a comparação entre as competências e habilidades da Indústria 4.0 versus as competências e habilidades dos acadêmicos, comparando o que está na literatura juntamente com os resultados obtidos na pesquisa de campo.

Quadro 2 - Literatura e resultados da pesquisa.

\begin{tabular}{|c|c|c|c|}
\hline AUTOR & ANO & CARACTERÍSTICAS & $\begin{array}{c}\text { PERCEPÇÃO DOS } \\
\text { ACADÊMICOS } \\
\end{array}$ \\
\hline $\mathrm{CNI}$ & 2016 & $\begin{array}{l}\text { Ter conhecimento multidisciplinar. } \\
\text { Ter elevado nível de conhecimento técnico. } \\
\text { Ter capacidade de interação com outras áreas do } \\
\text { conhecimento. }\end{array}$ & Características não identificadas. \\
\hline \multirow[b]{3}{*}{ WEF } & \multirow[b]{3}{*}{2016} & $\begin{array}{l}\text { Habilidades: } \\
\text { Cognitivas: flexibilidade cognitiva, criatividade, } \\
\text { raciocínio lógico, sensibilidade para problemas, } \\
\text { raciocínio matemático e visualização. } \\
\text { Físicas: força física e destreza manual e precisão. }\end{array}$ & $\begin{array}{l}\text { Habilidades identificadas: } \\
\text { flexibilidade cognitiva, raciocínio } \\
\text { lógico e sensibilidade para } \\
\text { problemas. }\end{array}$ \\
\hline & & $\begin{array}{l}\text { Competências básicas: } \\
\text { De conteúdo: aprendizagem ativa, expressão oral, } \\
\text { compreensão de leitura, expressão escrita e } \\
\text { formação em TIC. } \\
\text { De processo: escuta ativa, pensamento crítico, } \\
\text { monitoramento próprio e dos outros. }\end{array}$ & $\begin{array}{l}\text { Competências básicas } \\
\text { identificadas: pensamento crítico } \\
\text { e monitoramento próprio e de } \\
\text { outros. }\end{array}$ \\
\hline & & $\begin{array}{l}\text { Competências transversais: } \\
\text { Sociais: coordenação de equipe, inteligência } \\
\text { emocional, negociação, persuasão, orientação de } \\
\text { serviço, treinar e ensinar pessoas. } \\
\text { Sistêmicas: julgamento e tomada de decisão e } \\
\text { análise sistêmica. } \\
\text { Resolução de Problemas Complexos: capacidade } \\
\text { de resolver problemas complexos. } \\
\text { Gestão de Recursos: gestão de recursos } \\
\text { financeiros, gestão de recursos materiais, gestão de } \\
\text { pessoas e gestão de tempo. } \\
\text { Técnicas: manutenção e reparo de equipamentos, } \\
\text { operação e controle de equipamentos, } \\
\text { programação, controle de qualidade, design de } \\
\text { tecnologia e experiência de usuário e solução de } \\
\text { problemas. }\end{array}$ & $\begin{array}{l}\text { Competências transversais } \\
\text { identificadas: coordenação de } \\
\text { equipe, julgamento e tomada de } \\
\text { decisão e análise, capacidade de } \\
\text { resolver problemas complexos, } \\
\text { gestão de tempo e solução de } \\
\text { problemas. }\end{array}$ \\
\hline VILLIGER & $201-$ & $\begin{array}{l}\text { Ter formação multidisciplinar. } \\
\text { Ter conhecimento técnico para lidar com máquinas } \\
\text { inteligentes. } \\
\text { Ter habilidade para aprender e se adaptar. } \\
\text { Observar e tomar decisões. } \\
\text { Criatividade. }\end{array}$ & $\begin{array}{l}\text { Competências e habilidades } \\
\text { identificadas: observar e tomar } \\
\text { decisões. }\end{array}$ \\
\hline
\end{tabular}

Fonte: Elaboração própria a partir de dados da pesquisa.

\section{CONSIDERAÇÕES FINAIS}

Este estudo teve por objetivo identificar a percepção dos acadêmicos dos cursos de Administração e Comércio Exterior da Unesc, diante das suas competências e habilidades frente àquelas requeridas pela Indústria 4.0. 
Analisando o perfil dos acadêmicos das $7^{\mathrm{a}}$ e $8^{\mathrm{a}}$ fases dos cursos de Administração e Comércio Exterior da Unesc, identificou-se que, o curso de Administração é o que possui mais acadêmicos entrevistados, ambos os cursos apresentam mais acadêmicos do sexo masculino, e que a maioria deles residem na região da Amrec.

Após apresentar o perfil dos acadêmicos entrevistados, buscou-se identificar o conhecimento prévio dos acadêmicos sobre as características da Indústria 4.0. Em relação ao uso da tecnologia no ambiente pessoal, acadêmico e profissional, para a maior parte dos entrevistados, o uso da mesma é muito importante em todos os contextos; sobre os acontecimentos pertinentes a Indústria 4.0, descobriu-se que, os acadêmicos estão em contato com os conteúdos e notícias sobre a $4^{\mathrm{a}} \mathrm{RI}$, adquirindo conhecimento sobre o assunto.

Foi constatado que, os acadêmicos possuem conhecimento considerado baixo sobre as novas tecnologias trazidas pela Indústria 4.0; sobre o uso da nuvem para armazenamento de arquivos pessoais, acadêmicos ou profissionais. Ficou registrado que a maior parte utiliza pouco esse tipo de armazenamento, e maior parte concorda parcialmente que a Indústria 4.0 criará uma produção mais precisa e personalizável, ou seja, uma produção cada vez mais de acordo com o desejo do cliente.

Quanto ao conhecimento sobre os impactos, competências e habilidades da Indústria 4.0, notou-se que diante das competências e habilidades requeridas pela Indústria 4.0 frente as dos acadêmicos, os mesmos possuem em parte, cada característica descrita, ou seja, há a necessidade de desenvolvimento e aprimoramento das mesmas. Já em relação a criação de nova demanda por profissionais, os entrevistados concordam parcialmente, ou seja, nem todos concordam com o fato de que a Indústria 4.0 criará um novo mercado de trabalho, necessitando de novos profissionais hoje não existentes.

No que tange à mudança de capacitação profissional, a maioria dos entrevistados discordam que haverá necessidade de novos modelos de ensino e, que os moldes atuais estão de acordo com a proposta da Indústria 4.0. Já em relação aos meios utilizados para aquisição de conhecimento, os mais utilizados pelos acadêmicos foram a leitura de artigos, jornais, revistas ou livros, reportagens e palestras e, os menos utilizados para aquisição de conhecimento foram a partir de disciplinas ministradas nos cursos e outros meios, como por exemplo, pesquisa livres na internet.

No que diz respeito as profissões do Administrador, em relação ao grau de probabilidade de automatização, a profissão que mais se destacou no quesito de menos automatização, foi o gerente de recursos humanos, que de fato na Indústria 4.0 será mais valorizado, pois em um momento com grande automatização de processos, os recursos humanos da empresa devem ser 
melhores valorizados e capacitados, gerente de vendas tem probabilidade intermediária de automatização, segundo os entrevistados, e o profissional responsável por cálculos financeiros e fiscais, leva o grau mais alto de automatização.

A partir do contexto da pesquisa, buscou-se compreender se o acadêmico entrevistado se sente um futuro administrador preparado para atuar num ambiente da Indústria 4.0. A maior parte concordou que sim, que se sentem preparados para atuarem num ambiente totalmente novo, que até então era do conhecimento de poucos.

Dada a importância deste assunto, como proposta, sugere-se o estudo aprofundado sobre as características, tecnologias e inovações trazidas pela Indústria 4.0 por parte dos acadêmicos, para que os mesmos estejam preparados para o mercado de trabalho, que é desafiador para esta nova indústria. Sugere-se também a formação multidisciplinar do profissional, pois o mesmo atuará e realizará trabalhos em diversas áreas da empresa, sejam eles em equipes ou individual.

Desta forma, estudos futuros podem aprofundar o tema e compreender, a partir de uma amostra maior, bem como em outros cursos da instituição, e de outras universidades da região Sul e do Estado de Santa Catarina. Também sugere-se realizar um estudo sobre as matrizes curriculares dos cursos de Administração, em diversas regiões, para realizar a análise e comparar os conhecimentos ministrados pelos cursos em comparação aos conhecimentos trazidos pela Indústria 4.0, e verificar se ambos estão alinhados. Sugere-se ainda realizar um estudo com empresas da região para verificar o processo em que as mesmas se encontram em relação as tecnologias trazidas pela Indústria 4.0 e o seu processo de adaptação frente a $4^{\mathrm{a}}$ RI.

Em virtude dos fatos mencionados, percebe-se que o desenvolvimento deste tema possibilitou trazer, não somente dados atualizados e consistentes a respeito do nível de conhecimento dos acadêmicos em relação a Indústria 4.0 e suas características, mas identificar o nível do conhecimento dos acadêmicos sobre as fábricas inteligentes, inteligência artificial, big data, internet das coisas, a criação de novas demandas de profissionais, os impactos da Indústria 4.0 na profissão do Administrador, o nível de utilização da tecnologia e do armazenamento em nuvem de arquivos pessoais, acadêmicos e profissionais, os meios onde os conhecimentos sobre a $4^{\mathrm{a}}$ RI foram adquiridos, além de quais competências e habilidades requeridas pela Indústria 4.0 estão presentes no perfil destes acadêmicos. 


\section{REFERÊNCIAS}

AIRES, R. W. do A.; KEMPNER-MOREIRA, F.; FREIRE, P. de S. Indústria 4.0: competências requeridas aos profissionais da Quarta Revolução Industrial. In: CONGRESSO INTERNACIONAL DE CONHECIMENTO E INOVAÇÃO, Foz do Iguaçu. Anais [...]. 2017. Disponível em: https://proceeding.ciki.ufsc.br/index.php/ciki/issue/archive. Acesso em: 15 de set. 2020.

ASSOCIAÇÃO BRASILEIRA DE INTERNET INDUSTRIAL. A nova era da internet industrial. 2017. Disponivel em: https://www.abii.com.br/faq. Acesso em: 28 mar. 2018

CANÊDO, L. B. A revolução industrial. São Paulo: Atual, 2009.

CONFEDERAÇÃO NACIONAL DA INDÚSTRIA (Brasil). Desafios para a indústria 4.0 no Brasil. 2016. Disponível em: http://www.portaldaindustria.com.br/publicacoes/2016/8/des afios-para-industria-40-no-brasil. Acesso em: 24 mar. 2018.

CONFEDERAÇÃO NACIONAL DA INDÚSTRIA (Brasil). Relações trabalhistas no contexto da Indústria 4.0. 2017. Disponível em: http://www.portaldaindustria.com.br/relaco esdotrabalho/media/publicacao/chamadas/Relacoes_trabalhistas_web.pdf. Acesso em: 21 ago. 2018.

CONFEDERAÇÃO NACIONAL DA INDÚSTRIA (Brasil). Profissionais que dominarem competências da Indústria 4.0 vão ter mais chances no mercado. 2018. Disponível em: https://noticias.portaldaindustria.com.br/entrevistas/rafael-lucchesi/profissionais-quedominarem-competencias-da-industria-40-vao-ter-mais-chances-no-mercado/. Disponível em: 21 ago. 2018.

DIEHL, A. A.; TATIM, D. C. Pesquisa em ciências sociais aplicadas: métodos e técnicas, São Paulo, Pearson Prentice Hall, 2004.

DREHER, A. The Smart Factory of the Future - Part 1. Belden News. Disponível em; http://www.belden.com/blog/industrialethernet/The-Smart-Factory-of-theFuture-Part-1.cfm. Acesso em: 28 mar. 2018.

DUARTE, S. V.; FURTADO, M. S. V. Trabalho de conclusão de curso (TCC) em ciências sociais aplicadas. São Paulo: Saraiva, 2014.

MATTAR, F. N. Pesquisa de Marketing. 3ª ed. São Paulo, Atlas, 2001.

MICHEL, M. H. Metodologia e pesquisa científica em ciências sociais: um guia prático para acompanhamento da disciplina e elaboração de trabalhos monográficos. São Paulo: Atlas, 2015.

RICHARDSON, R. J. Pesquisa social: métodos e técnicas. São Paulo: Ed. Atlas, 1985.

SCHWAB, K. A quarta revolução industrial. São Paulo, Edipro, 2016.

SOUZA. E. S. de. Características e impactos da Indústria 4.0: percepção de estudantes de ciências contábeis. 2018. Monografia (Bacharel em Ciências Contábeis, Universidade Federal de Santa Catarina, Florianópolis. Disponível em: https://repositorio.ufsc.br/handle/123456789 /188365. Acesso em: 14 ago. 2018. 
UNIVERSIDADE DO EXTREMO SUL CATARINENSE. Administração de empresas. 2018. Disponível em: http://www.Unesc.net/portal/capa/index/40. Acesso em: 12 out. 2018.

Villiger, P. Qualificação para a Indústria 4.0: uma solução da indústria para a indústria. (201?). Disponível em: http://www.senaipr.org.br/simposio-industria40/uploadAddress/FEST O[72923].pdf. Acesso em: 21 ago. 2018.

WORLD ECONOMIC FORUM. The future of jobs: employment, skills and workforce strategy for the fourth industrial revolution. 2016. Disponível em: http://www3.weforum.org/ docs/WEF_Future_of_Jobs.pdf. Acesso em: 21 ago. 2018. 